\title{
15 years of methods
}

\author{
We celebrate the 15th anniversary issue of Nature Methods with a look back on the past decade and a half, and a \\ look forward to the future of methods development.
}

n several cultures, turning 15 represents an important milestone, a coming-ofage. For some of us who have been with Nature Methods since its early days as a nascent journal, it is hard to fathom just how much has changed in life sciences research.

Fifteen years ago, a human genome had only recently been mapped for the first time, at enormous effort and expense - there was no such technique as high-throughput genome sequencing. Cell biologists were squinting at hazy cellular structures in fluorescent micrographs - broadly applicable methods for super-resolution microscopy had not yet been developed. Deep learning was an idea far ahead of its time, with computer hardware nowhere near powerful enough to meet the demands of such algorithms. Those trying to make sense of protein blobs using cryo-electron microscopy were still years away from determining structures at atomic resolution. And the noun form of 'crisper' only referred to a refrigerator compartment for storing fruits and vegetables.

Back in 2004, as editors of a newly established journal, we worried about how we could evolve and what we would publish when the major fields we were covering reached maturity. Fast forward to 2019 and we are glad to report that the pace of methodological development across basic life sciences research shows no signs of slowing down. Over the years we have witnessed the emergence of whole new fields based on methodological breakthroughs, including several that we have celebrated in our yearly Method of the Year feature, such as optogenetics, single-cell sequencing, lightsheet imaging and induced pluripotent stem cells. Other already-established techniques, including genome engineering, cryoelectron microscopy and mass spectrometry, have been reenergized by breakthrough technological developments.

While many fields have undergone periods of rapid new methodological development, it is true that methods in other areas have indeed reached maturity. As a result, the scientific content of an issue of Nature Methods today looks totally different to an issue from 2004. However, our guiding principle outlined in our very first editorial in October 2004 remains the same: "Nature Methods will make use of the periodical format to introduce methods at a stage where they have not yet gained a track record of success but show definite promise and are likely to stimulate other researchers."

Over the years we have introduced new formats to meet the needs of the methods development community, including Analysis and Resource. The way we peer review papers has also evolved, as we introduced an option for double blind peer review and began working with Code Ocean to facilitate the review of computational code. We now list editor's names on the papers we publish, to promote transparency and highlight the behind-the-scenes work that we do in selecting and curating research to communicate to our audience. We strengthened our standards for reproducibility by introducing a statistical reporting summary, requiring data availability statements and mandating data deposition to public repositories where applicable.

Our approach to communicating with our readers has also changed. In 2004, social media was all about blogging and Twitter did not yet exist. We are now quite active on Twitter (@naturemethods) and plan to be even more so in the future, highlighting not only our own content, but also general scientific news that we think will be interesting to the communities that we serve. Our old Methagora blog has been retired, but you can still find the older content plus much new content on a modern, community-based site in which Nature Methods participates, the Springer Nature Protocols and Methods Community. To help celebrate this special anniversary, we will include a blog post that features some of the fabulous cover illustrations that have graced the front of Nature Methods issues and give some insights into how our covers are chosen and developed.

In this special issue, we invited 39 researchers that we consider to be methodological visionaries to give their views on what future methods developments are needed to address the most interesting and important challenges in their respective fields. Our aim was to gather a diverse set of voices from across a broad swath of research areas, and yet, it is intriguing to note several convergent themes. Many of the researchers we queried mentioned the need for synergies between different technologies to achieve a deeper understanding of complex systems like the brain. Another common theme, mentioned by researchers from the genomics, proteomics, imaging and structural biology fields, was the desire to map the fine spatial details of cellular organization at high resolution. It is also apparent from reading the comments that advanced computational algorithms, especially those based on machine learning, are poised to make an even greater impact across multiple areas of life science research.

Of course, these views represent just a small slice of the interesting research happening across the life sciences. We are more than happy to hear visions from other researchers as well, and will collect and post selected contributions on our community page. Please send an e-mail to chief editor Allison Doerr (a.doerr@us.nature.com) with the subject line 'Voices in methods development'.

An anniversary issue is also a fitting opportunity to welcome the two newest members of our team, Arunima Singh and Lin Tang (who joins us with three years' experience at Nature Communications). Our new editors join existing team members Nina Vogt, Rita Strack, Lei Tang and journalist Vivien Marx. And, sadly, we are saying goodbye to long-time editor Nicole Rusk, who is returning to her academic roots - we wish her all the best.

Finally, we need to give a huge thank you to you - our authors, reviewers and readers. The journal could not have achieved its current level of success without your tremendous and continuing support. Please join us in a toast to 15 great years - and here's to many more!

Published online: 27 September 2019 https://doi.org/10.1038/s41592-019-0594-5 